\title{
Changes in the redox state and composition of the quinone pool of Escherichia coli during aerobic batch-culture growth
}

\begin{abstract}
Correspondence
M. J. Teixeira de Mattos

teixeira@science.uva.nl
\end{abstract}

Received 31 January 2007

Accepted 2 February 2007

\author{
M. Bekker, ${ }^{1}$ G. Kramer, ${ }^{2}$ A. F. Hartog, ${ }^{3}$ M. J. Wagner, ${ }^{4}$ C. G. de Koster, ${ }^{2}$ \\ K. J. Hellingwerf ${ }^{1}$ and M. J. Teixeira de Mattos ${ }^{1}$
}
${ }^{1}$ Molecular Microbial Physiology Group, Swammerdam Institute for Life Sciences, BioCentrum, University of Amsterdam, Nieuwe Achtergracht 166, 1018 WV Amsterdam, The Netherlands
${ }^{2}$ Biological Mass-Spectrometry Group, Swammerdam Institute for Life Sciences, BioCentrum, University of Amsterdam, Nieuwe Achtergracht 166, 1018 WV Amsterdam, The Netherlands
${ }^{3}$ Biomolecular Synthesis Group, Van't Hoff Institute for Molecular Sciences, Nieuwe Achtergracht 129, 1018 WS Amsterdam, The Netherlands
${ }^{4}$ Molecular Cell Physiology Group, Institute of Molecular Cell Biology, Vrije Universiteit, De Boelelaan 1087, 1081 HV Amsterdam, The Netherlands

\begin{abstract}
Ubiquinones (UQs) and menaquinones (MKs) perform distinct functions in Escherichia coli. Whereas, in general, UQs are primarily involved in aerobic respiration, the MKs serve as electron carriers in anaerobic respiration. Both UQs and MKs can accept electrons from various dehydrogenases, and may donate electrons to different oxidases. Hence, they play a role in maintaining metabolic flexibility in E. coli whenever this organism has to adapt to conditions with changing redox characteristics, such as oxygen availability. Here, the authors report on the changes in both the size and the redox state of the quinone pool when the environment changes from being well aerated to one with low oxygen availability. It is shown that such transitions are accompanied by a rapid increase in the demethylmenaquinone pool, and a slow increase in the MK pool. Moreover, in exponentially growing cultures in a well-shaken Erlenmeyer flask, it is observed that the assumption of a pseudo-steady state does not hold with respect to the redox state of the quinone pool.
\end{abstract}

\section{INTRODUCTION}

The quinones serve as membrane-associated electron carriers in the respiratory chain that carry out electron transfer between various electron donors and acceptors. Ubiquinone (UQ)-8 (coenzyme $\mathrm{Q}_{8}$ or $\mathrm{UQ}_{8}$ ) is the main quinone in Escherichia coli involved in aerobic respiration, and in electron transfer towards nitrate (Brondijk et al., 2002; Soballe \& Poole, 1999; Wallace \& Young, 1977). Under anoxic conditions, but in the presence of other electron acceptors, either demethylmenaquinone (DMK)-8 $\left(\mathrm{DMK}_{8}\right)$ or menaquinone $(\mathrm{MK})-8\left(\mathrm{MK}_{8}\right)$ serves as the main electron-transferring quinone. Whether the cell uses $\mathrm{DMK}_{8}$ or $\mathrm{MK}_{8}$ depends on the particular carbon source and electron acceptor present in the growth medium (Unden, 1988; Wissenbach et al., 1992).

It is well-known that $E$. coli is endowed with a highly versatile respiratory chain that allows it to adapt rapidly to conditions that may vary to a great extent with respect to

Abbreviations: DMK, demethylmenaquinone; MK, menaquinone; UQ, ubiquinone. their redox state (de Graef et al., 1999; Poole \& Cook, 2000; Smith \& Neidhardt, 1983). In order to achieve this flexibility, synthesis and turnover of both UQs and MKs should be rapid upon changing conditions. It has been shown that control of the composition of the quinone pool does not take place at a transcriptional or translational level, but rather occurs at the post-translational level of the access enzymes in the $\mathrm{MK}_{8}$ biosynthetic pathway (Shestopalov et al., 1997). This form of regulation implies that $\mathrm{UQ}_{8}$ and $\mathrm{MK}_{8}$ production can be effected within minutes. Our previous work has elucidated some of the subtleties that underlie the control of electron flow through the alternative pathways of the respiratory chain (Alexeeva et al., 2002; Calhoun et al., 1993). Here, we present data that illustrate the sensitivity of the chain to external redox conditions by use of a single and rapid method that determines the content of the quinone pools and the redox state of the UQ pool. It is shown that the assumption of a pseudo-steady state during the exponential growth phase in a standard experimental set-up with a rotary shaker is not correct with respect to the composition and functioning of the respiratory chain. The synthesis of 
$\mathrm{DMK}_{8}$ during transitions from high to low oxygen tensions is discussed.

\section{METHODS}

Strains and growth conditions. E. coli strain MC4100 was used throughout this study. A $\Delta u b i E$ mutant derived from JC7623, and called JC7623 $\Delta 4-1$, which was kindly provided by C. Clarke (Lee et al., 1997), was also used. Cells in batch cultures were grown in Evans medium (Evans et al., 1970) ( $\mathrm{pH} 6.9 \pm 0.1$ ) at $37{ }^{\circ} \mathrm{C}$. Sodium phosphate was used as a buffer at a concentration of $100 \mathrm{mM}$. Glucose, selenite and thiamine were added to final concentrations of $50 \mathrm{mM}, 30 \mu \mathrm{g}^{-1}$ and $15 \mathrm{mg} \mathrm{l}^{-1}$, respectively. Aeration was accomplished by growing $30 \mathrm{ml}$ culture volumes in $300 \mathrm{ml}$ Erlenmeyer flasks, or $100 \mathrm{ml}$ volumes in 11 Erlenmeyer flasks, in a rotary shaker (Gallenkamp Orbital Incubator) set at 200 r.p.m. Cells were taken from cultures grown aerobically overnight, and inoculated at an $\mathrm{OD}_{600}$ of 0.1 .

Cells grown in chemostat conditions were grown under glucoselimited conditions (Bioflo III) at a constant dilution rate of $0.35 \pm 0.01 \mathrm{~h}^{-1}$ in a vessel containing $1200 \mathrm{ml}$ culture. Glucose $(10 \mathrm{mM})$ was used as the single carbon and energy source. Selenite and thiamine were added to final concentrations of 30 and $15 \mathrm{mg} \mathrm{l}^{-1}$, respectively. $\mathrm{pH}$ was maintained at $7.0 \pm 0.1$ by titrating with sterile $1 \mathrm{M} \mathrm{NaOH}$, and temperature was set to $35{ }^{\circ} \mathrm{C}$. Oxygen supply was regulated by a Brooks mass flow controller $5850 \mathrm{E}$ series. The air

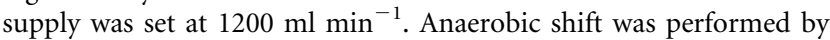
changing the input gas from air to nitrogen. Residual dissolved oxygen was monitored by an Ingold polarographic oxygen sensor, with a detection limit of $100 \mathrm{nM}$.

Quinone extraction and analysis. Samples from chemostat cultures were taken by use of a sampling device (Lange et al., 2001) to ensure reproducible and quantitative aliquots within $0.5 \mathrm{~s}$. Samples from batch cultures were taken by pipetting within $2 \mathrm{~s}$. Samples $(2 \mathrm{ml})$ were quenched with $6 \mathrm{ml}$ ice-cold $0.2 \mathrm{M} \mathrm{HClO}_{4}$ in methanol. Petroleum ether $\left(6 \mathrm{ml} ; 40-60{ }^{\circ} \mathrm{C}\right)$ was then added rapidly to the mixture, and vortexed for $1 \mathrm{~min}$. After the mixture was centrifuged (900 $\mathrm{g}, 2 \mathrm{~min}$ ), the upper petroleum ether phase was removed, transferred to a test tube, and evaporated to dryness under a flow of nitrogen. Another $6 \mathrm{ml}$ petroleum ether was added to the lower phase, and the vortexing and centrifugation steps were repeated. The upper phases were combined. After evaporation to dryness, extracts could be stored for at least 7 days under nitrogen at $-20{ }^{\circ} \mathrm{C}$ without any detectable auto-oxidation. Immediately before use, the extracted UQ/ ubiquinol was resuspended with a glass rod in $80 \mu \mathrm{l}$ ethanol and analysed in a HPLC system (Pharmacia LKB gradient pump 2249 system, with an LKB 2151 variable-wavelength monitor) containing a reverse-phase Lichrosorb (Chrompack) $10 \mathrm{RP} 18$ column $(4.6 \mathrm{~mm}$ i.d., $250 \mathrm{~mm}$ length). The column was equilibrated with ethanol : methanol $(1: 1, \mathrm{v} / \mathrm{v})$, and this mixture was used as the mobile phase. The flow rate was set at $1 \mathrm{ml} \mathrm{min}{ }^{-1}$. Detection of the quinones was performed at $290 \mathrm{~nm}$ for UQs, at $248 \mathrm{~nm}$ for MKs, and at $270 \mathrm{~nm}$ to record all quinones simultaneously. The amounts of all quinones were calculated from the peak areas using $\mathrm{UQ}_{10}$ and $\mathrm{MK}_{4}$ as standards, according to the method applied by Shestopalov et al. (1997). Methanol, ethanol and petroleum ether were of analytical grade.

Quinone identification. Peaks were identified by UV/Vis spectral analysis and mass spectral analysis. A UV/Vis spectrum of $\mathrm{DMK}_{8}$ was kindly provided by Dr Bogachev (Moscow State University, Russia). For mass spectral analysis, fractions collected from the HPLC were evaporated under nitrogen, and redissolved into $89 \%$ acetonitrile, $10 \%$ water and $1 \%$ formic acid (v/v; LC-Grade; Merck). The fractions were analysed by off-line nano-electrospray MS using coated
PicoTips (Econo12; New Objective) on an electrospray ionization QTOF mass spectrometer (Micromass). Ions selected for MS/MS collided with argon in the hexapole collision cell. Data processing and evaluation were performed using Masslynx software (Micromass).

\section{RESULTS}

\section{Method for determination of the reduction state and content of the quinone pools}

A method that has been used to determine the reduction state of the ubiquinone pool in Paracoccus denitrificans (Otten, 2001; Van den Bergen et al., 1994) was adapted for determining the reduction state of the quinone pool in $E$. coli. Quinones were isolated as described (see Methods), and separated by HPLC. The UV/Vis spectra of all peaks shown in the chromatogram (Fig. 1) were recorded (shown at $270 \mathrm{~nm}$ ). The peaks at 8 and 12.5 min elution times showed spectra typical for $\mathrm{UQ}_{8} \mathrm{H}_{2}$ and $\mathrm{UQ}_{8}$. Chromatograms of quinone isolations from the $\Delta u b i E$ mutant, which is unable to catalyse the final methylation step for formation of UQ (Lee et al., 1997; Wissenbach et al., 1992), did not show these two peaks (Fig. 1). Mass spectral analysis showed the presence of the correct masses for $\mathrm{UQ}_{8} \mathrm{H}_{2}$ and $\mathrm{UQ}_{8} \quad(729.5$ and $727.5 \mathrm{~m} / z$, respectively). In addition, MS/MS fragmentation of selected UQ peaks showed tropylium ions at $197.1 \mathrm{~m} / \mathrm{z}$. Formation of these tropylium ions is specific for fragmented UQs (Geyer et al., 2004).

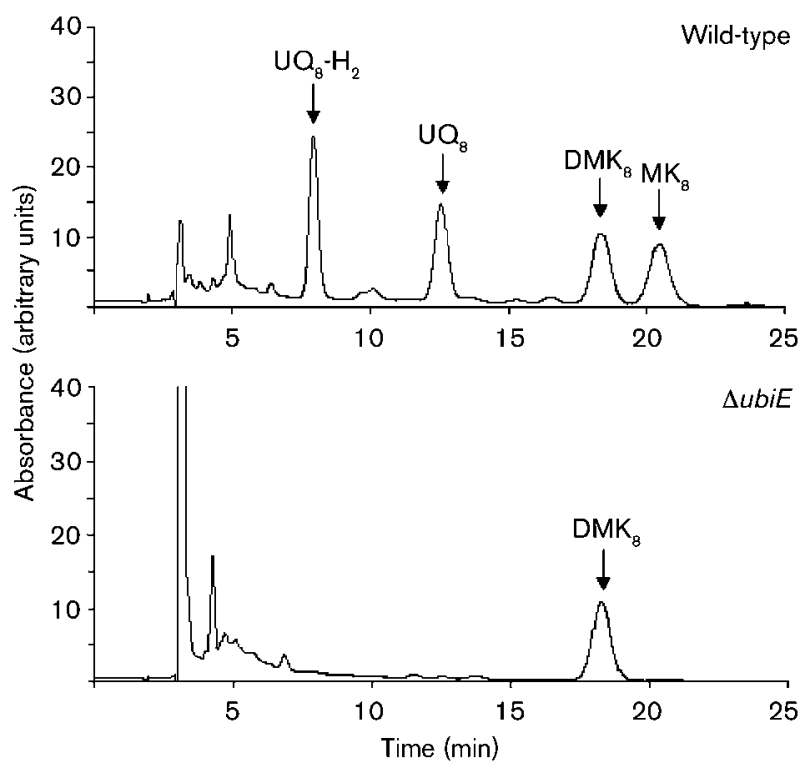

Fig. 1. HPLC analysis of the quinone pool of $E$. coli. Extracts from an E. coli MC4100 (wild-type) and JC7623 $\Delta 4-1$ ( $\Delta u b i E$ ) culture were fractionated by reverse-phase HPLC. The mixture of quinones was analysed by UV/Vis absorption spectroscopy at $270 \mathrm{~nm}$ (see Methods). 
The peaks at 18 and at $21 \mathrm{~min}$ showed UV/Vis spectra typical for $\mathrm{DMK}_{8}$ (reference spectrum kindly provided by Dr Bogachev) and $\mathrm{MK}_{4}$. Mass spectral analysis showed the presence of the masses of $\mathrm{DMK}_{8}$ and $\mathrm{MK}_{8}$ (703.6 and 717.6 $\mathrm{m} / \mathrm{z}$, respectively). Confirmation by MS/MS (173.1 and $187.1 \mathrm{~m} / z$ for $\mathrm{DMK}_{8}$ and $\mathrm{MK}_{8}$, respectively; Geyer et al., 2004) showed the expected fragmentation products. Quinone samples isolated from the $\Delta u b i E$ mutant (Lee et al., 1997), which is also impaired in the methylation of DMK to MK, did show a peak at 18 , but not at $21 \mathrm{~min}$. From these data, it can be concluded that the peaks at 18 and 21 min are due to the presence of $\mathrm{DMK}_{8}$ and $\mathrm{MK}_{8}$, respectively, and that the peaks identified in Fig. 1 consist solely of the indicated quinones. Peaks for reduced $\mathrm{DMK}_{8}$ and $\mathrm{MK}_{8}$ were found to elute around 6 and $7 \mathrm{~min}$, respectively (data not shown). These reduced forms, however, always showed nearly complete oxidation during quinone isolation, and are therefore not included in this report. Hence, it is assumed that the determined $\mathrm{DMK}_{8}, \mathrm{MK}_{8}$, represents the total of oxidized plus reduced $\mathrm{DMK}_{8}$, i.e. the total of oxidized plus reduced $\mathrm{MK}_{8}$.

\section{Effect of the length of the sampling time: kinetics of $\mathrm{DMK}_{\mathbf{8}}$ production}

When the size of the UQ pool is known (in moles per cell mass), together with the respiration rate (in moles of oxygen reduced per unit time per cell mass), the turnover of the UQ pool can be calculated. Thus, in rapidly aerobically growing $E$. coli cells $\left(\mu 0.7 \mathrm{~h}^{-1}\right)$ with a specific respiration rate of $10 \mathrm{mmol} \mathrm{h}^{-1} \mathrm{~g}^{-1}$ (Calhoun et al., 1993), and a UQ content of $1 \mu \mathrm{mol} \mathrm{g}^{-1}$ (see Fig. 6), the turnover of the $\mathrm{UQ}_{8}$ pool is approximately $180 \mathrm{~ms}$. Hence, fast

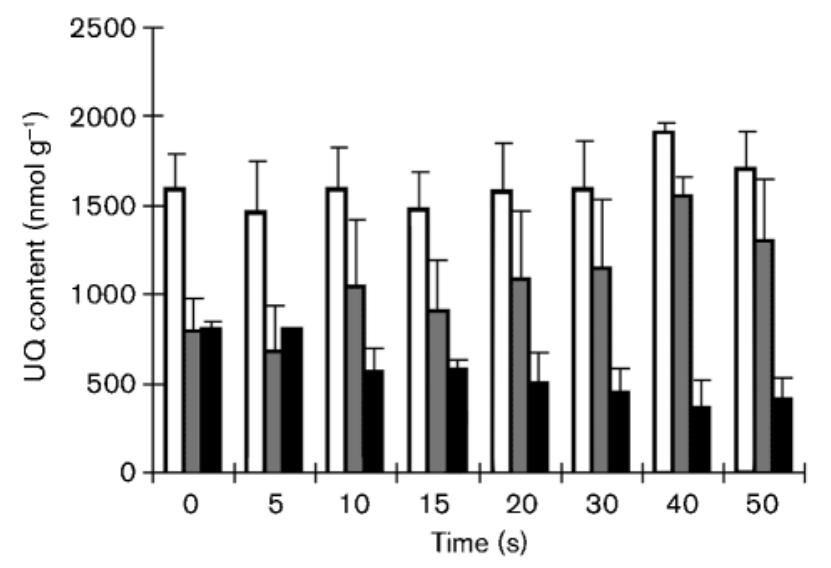

Fig. 2. The effect of restricted oxygen availability on the reduction state of the $\mathrm{UQ}_{8} / \mathrm{UQ}_{8} \mathrm{H}_{2}$ pool of $E$. coli. Samples were directly quenched in a methanol/ $\mathrm{HClO}_{4}$ solution to minimize alterations during the isolation procedure. The $x$-axis shows the time points of sampling after a shift to low aeration following aerobic growth to an $\mathrm{OD}_{600}$ of 3.0. White bars, total $\mathrm{UQ}_{8}+\mathrm{UQ}_{8} \mathrm{H}_{2}$; grey bars, $\mathrm{UQ}_{8} \mathrm{H}_{2}$; black bars, $\mathrm{UQ}_{8}$. Error bars indicate SD. quenching of the sampled cells is essential to obtain representative data regarding the redox state of these pools. The redox state of the quinone pool was analysed in time, with a frequency of $5 \mathrm{~s}$, when a mid-exponential culture with a biomass concentration of $1 \mathrm{~g}$ dry weight $1^{-1}$ was transferred from aerobic well-shaken conditions to conditions without shaking (i.e. the oxygen supply was limited by the rate of oxygen diffusion). The redox state of the UQ pool remained constant for at least $5 \mathrm{~s}$, since no increase in amount of $\mathrm{UQ}_{8} \mathrm{H}_{2}$ was observed in the first $5 \mathrm{~s}$. However, within $10 \mathrm{~s}$, the $\mathrm{UQ}_{8}$ pool showed a significant reduction that lasted until $40 \mathrm{~s}$ after limitation of the oxygen supply (Fig. 2). Care was taken to quench all further samples within $5 \mathrm{~s}$.

Over the analysis period, the total content of $\mathrm{UQ}_{8}$ did not change significantly upon restriction of the oxygen supply. In contrast to this, the $\mathrm{DMK}_{8}$ content showed a large increase during the first $40 \mathrm{~s}$ (Fig. 3). No significant increase in $\mathrm{MK}_{8}$ content was observed over the analysis period; however, during fully anaerobic conditions, both the $\mathrm{DMK}_{8}$ and the $\mathrm{MK}_{8}$ content are known to be high (Unden, 1988).

\section{Effect of a shift to anaerobiosis in controlled carbon-limited chemostat conditions}

\section{Kinetics of $\mathbf{M K}_{\mathbf{8}}$ production}

To determine the kinetics of $\mathrm{MK}_{8}$ synthesis, analysis after a shift to anaerobiosis was performed over a longer time frame. For this, growth was performed aerobically in a glucose-limited chemostat at a dilution rate of $0.35 \mathrm{~h}^{-1}$, allowing long sampling times upon a shift to anaerobiosis, without disturbance of the culture. Complete anaerobiosis was achieved by sparging nitrogen into the culture, resulting in a decrease of dissolved oxygen tension (dOT) values below the sensitivity of the oxygen electrode after $3 \mathrm{~min}$. Samples for quinone analysis were quenched using a

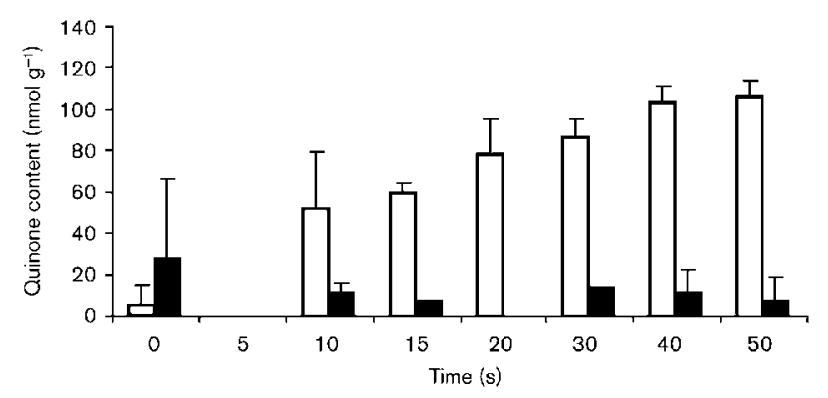

Fig. 3. In E. coli, restricted oxygen availability induces a fast production of $\mathrm{DMK}_{8}$. Samples were directly quenched in a methanol $/ \mathrm{HClO}_{4}$ solution to minimize alterations during the isolation procedure. The $x$-axis shows the time of sampling after a shift to low aeration. At $5 \mathrm{~s}$, the amounts of $\mathrm{DMK}_{8}$ and $\mathrm{MK}_{8}$ were below the detection limit $\left(1 \mathrm{nmol} \mathrm{g}{ }^{-1}\right)$. White bars, $\mathrm{DMK}_{8}$; black bars, $\mathrm{MK}_{8}$. Error bars indicate SD. 
rapid sampler, which is designed to quench cells within 0.5 s (Lange et al., 2001). As can be seen from Fig. 4, the transition resulted in a rapid reduction of the UQ pool from $10 \%$ to approximately $40 \%$, followed by a reoxidation to a level of about $25 \% . \mathrm{DMK}_{8}$ synthesis occurred rapidly after the transition, at an initial rate quite similar to that observed in exponential non-limited cultures (Fig. 3). Synthesis started within $1 \mathrm{~min}$ after nitrogen sparging, whereas oxygen was not completely depleted at that time point (Fig. 4). As with the batch cultures, this illustrates that $\mathrm{DMK}_{8}$ synthesis can take place in the presence of oxygen. Further, the rapid onset of synthesis suggests the presence of the synthesis pathway under fully aerobic conditions. This supports the conclusions of Shestopalov et al. (1997) who used an inhibitor of translation to show that the biosynthetic machinery is always present under aerobic conditions, and is activated under anaerobic conditions.

Whereas DMK synthesis was initiated within $10 \mathrm{~s}$ after the shift to anaerobiosis, and DMK levels reached a maximum within $1 \mathrm{~min}$ (Fig. 3), the increase in the total MK content was lower and much more gradual, with levels appearing to rise slowly, but continually, over the entire $15 \mathrm{~min}$ measurement period (Figs 3 and 4 ). Note that $S$-adenosylmethionine : 2-demethylmenaquinone methyltransferase (the $u b i E$ gene product methylating DMK to MK) should be present under these conditions (Shestopalov et al., 1997).

Redox state of the UQ pool during exponential growth During exponential growth on glucose, the specific respiration rate of $E$. coli is relatively high (typically 8-10 mmol oxygen $\mathrm{h}^{-1}$ (g dry weight) ${ }^{-1}$; e.g. Calhoun et al., 1993). Provided sufficient oxygen is available to the cells, under these conditions a (pseudo-)steady state is generally presumed to exist with respect to cellular composition, energy and redox state. In growth medium in which the stationary phase is brought about by exhaustion of the electron donor, one would expect the UQ pool to become largely oxidized upon entry of the cells into stationary phase. The rapid changes observed, and the high sensitivity of the respiratory chain with respect to the availability of oxygen, urged us to test this assumption. It was observed that in an experimental set-up that is representative of the common practice of closed exponential growth experiments, the percentage of reduced $\mathrm{UQ}_{8}$ increased during the exponential phase of growth, suggesting that electron transfer to oxygen is impeded by the limited availability of oxygen (see Fig. 5). It is noteworthy that the increase occurred from $\mathrm{OD}_{600}$ values as low as 0.3 , which is equivalent to a biomass concentration of approximately $0.1 \mathrm{~g}$ dry weight $\mathrm{l}^{-1}$, and at dissolved oxygen percentages as
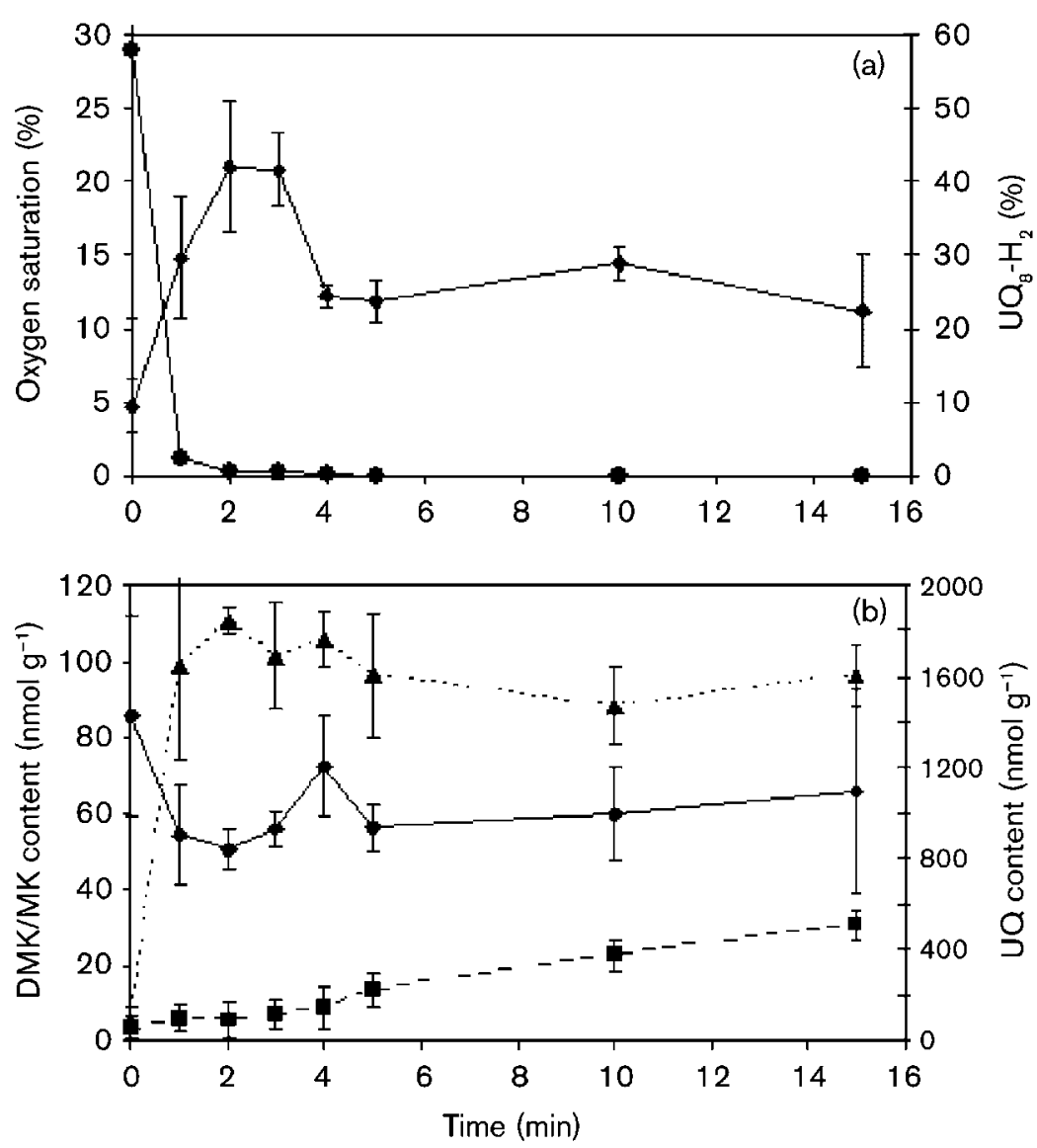

Fig. 4. $\mathrm{MK}_{8}$ and $\mathrm{DMK}_{8}$ synthesis, percentage $\mathrm{UQ}_{8} \mathrm{H}_{2}$, and percentage oxygen saturation, upon a shift to anaerobiosis in controlled chemostat conditions. Samples were quenched using a fast sampler, which ensures that quenching times are below $0.5 \mathrm{~s}$. The $x$-axis shows the time of sampling after a shift to anaerobiosis; $t=0$ is the time of shift to anaerobiosis. (A) $\diamond, \mathrm{UQ}_{8} \mathrm{H}_{2} ; \bullet$, oxygen saturation. (B) $\boldsymbol{\Delta}, \mathrm{DMK}_{8} ; \mathbf{\square}, \mathrm{MK}_{8} ; \boldsymbol{\cup} \mathrm{UQ}_{8}$. Error bars indicate SD. 
high as $80 \%$. These dry weights are comparable to amounts used routinely to circumvent artefacts due to a high cell concentration (Constantinidou et al., 2006). Interestingly, the increase in redox state of the UQ pool was mainly caused by a decrease in the oxidized UQ content (data not shown), whilst the reduced UQ content was stable throughout growth. The cellular $\mathrm{UQ}_{8} \mathrm{H}_{2}$ content was constant during exponential growth, but showed a decrease in the stationary phase. As shown in Fig. 5, true exponential growth was observed throughout the growth phase, with a constant doubling time of $0.9 \pm 0.05 \mathrm{~h}$. According to expectations, the UQ pool became more oxidized upon entry into stationary phase, which coincides with glucose depletion as measured routinely by HPLC (data not shown). Cells grown in nitrogen-limited medium showed similar temporal alterations in the redox state of the UQ pool (data not shown). These latter cells continue to oxidize glucose during the stationary phase, but at a much lower rate (see Discussion).

\section{Quinone content during exponential growth}

In a separate experiment, the amounts of the various quinone types were quantified during exponential growth. The data are shown in Fig. 6. UQ is known to be the most abundant quinone during aerobic growth of $E$. coli. Shestopalov et al. (1997) reported a UQ content of 300 $\mathrm{nmol}$ ( $\mathrm{g}$ dry weight cells) ${ }^{-1}$ during mid-exponential growth. The amounts measured in our experiments were up to almost 10-fold higher, particularly at the onset of exponential growth. It cannot be excluded that these differences are due to differences in growth medium (simple salts versus Luria broth) and/or the E. coli strain used (MC4100 versus ECL942), but it is noteworthy that the UQ content decreased continuously (to about $20 \%$ of the initial content) throughout the exponential phase. In the earlier report by Shestopalov et al. (1997), cells were

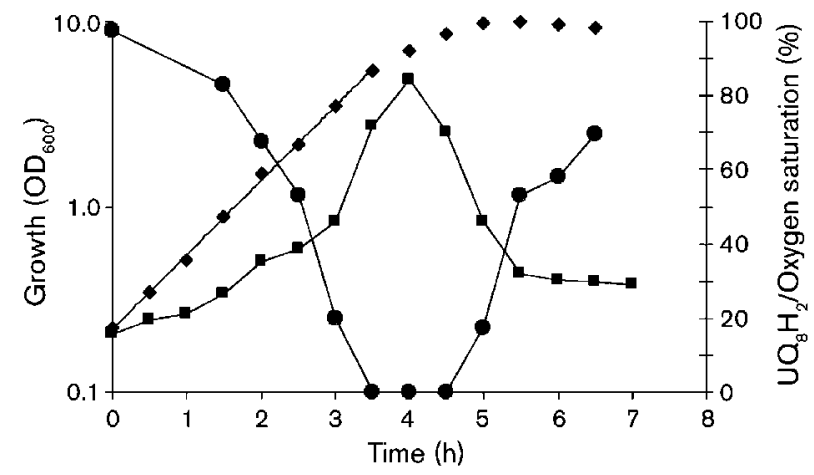

Fig. 5. Growth-phase dependence of the redox state of the $\cup_{8}$ pool of $E$. coli during growth in minimal medium with glucose. The reduction state of the $\mathrm{UQ}_{8}$ pool was measured in minimal medium supplied with $50 \mathrm{mM}$ glucose in shake cultures. $\mathbf{\square}, \mathrm{UQ}_{8} \mathrm{H}_{2} ; \bullet$, oxygen saturation; $\mathrm{OD}_{600}$. Data are from a single representative experiment.

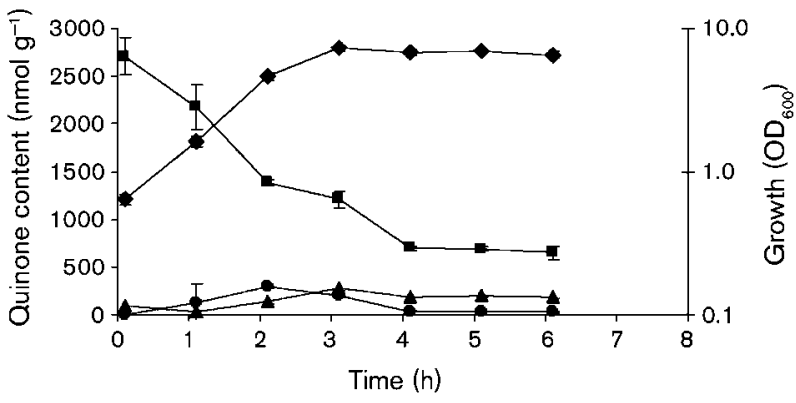

Fig. 6. Biomass-specific quinone content during growth in minimal medium with glucose. The composition of the quinone pools was measured in minimal medium supplied with $50 \mathrm{mM}$ glucose in shake cultures. $\mathbf{\square}$, total $\cup Q_{8}$ (oxidized + reduced); $\mathrm{DMK}_{8} ; \boldsymbol{\Delta}, \mathrm{MK}_{8} ;, \mathrm{OD}_{600}$. Error bars indicate SD.

collected by centrifugation before assessment of the quinone content. Together, these findings may well explain the observed differences. In agreement with Shestopalov et al. (1997), it was observed that $\mathrm{DMK}_{8}$ was present under the conditions tested. Its presence showed a strong decrease upon entry into stationary phase.

\section{DISCUSSION}

Organisms like E. coli show an enormous metabolic flexibility that allows them to proliferate in many different environments, including with respect to carbon sources and the availability and nature of external electron acceptors, on the one hand, and to cope with sudden changes in many physicochemical parameters, such as nutrient concentrations, osmolarity, $\mathrm{pH}$ and temperature, on the other. The respiratory chain constitutes a major catabolic module that brings this flexibility about (Neijssel \& Teixeira de Mattos, 1994). This is due first to the respiratory chain being branched, which allows for a variable efficiency of free energy conservation (Calhoun et al., 1991, 1993; Calhoun \& Gennis, 1993), and second to the potential to express a wide range of electrontransferring mediators and redox enzymes with different redox potentials and specificities, respectively (Berg \& Stewart, 1990; Cole, 1996). Hence, a broad range of external electron acceptors can be used by the organism.

Quantitative studies on functional, regulatory and structural aspects of the respiratory chain demand well-defined and well-controlled experimental set-ups in which the availability of both electron donors and acceptors is either at a constant subsaturation level, or saturated throughout the experiment. Whereas the former condition can be obtained by the use of chemostats (Alexeeva et al., 2000; Hommes et al., 1991), the latter is usually presumed to occur in well-shaken batch cultures grown under standard conditions. It is common practice to consider a culture that shows exponential growth to be in a steady state with 
respect to its macromolecular composition and enzymic make-up on the one hand, and with respect to nutrient availability on the other. We have shown, however, that this is not the case for E. coli grown under the standard batch conditions mentioned above. In order to quantify the regulation of the composition and redox state of the respiratory chain, a sensitive method was developed to determine the nature and content, as well as the redox state of the quinone pools. Optimization of the HPLC-based analysis methodology introduced by Shestopalov et al. (1997) and Unden (1988) resulted in sufficient resolution to discriminate the reduced and oxidized forms of UQ, MK and DMK at the nanomole per gram dry weight level. In addition, it is shown that the use of rapid sampling techniques to quench metabolism within a few seconds is paramount to obtain representative data because the redox state of the UQ changes significantly beyond this time window upon changes in stirring rate. In addition, the results presented here further illustrate the need for such rapid techniques even for cells that are not provided sufficient oxygen start to accumulate $\mathrm{DMK}_{8}$.

Our analysis shows clearly that although exponential growth does occur in a batch culture that is aerated by orbital shaking, dramatic changes occur during this growth phase with respect to the redox state and the absolute content of UQ. Whereas, initially, in low-density cultures, the redox state of UQ was found to be the same as determined for highly aerated cultures, such as chemostat cultures $\left(10 \% \mathrm{UQH}_{2}\right)$, or controlled batch cultures equipped with baffles and forced airflow $\left(40 \% \mathrm{UQH}_{2}\right.$, data not shown), the UQ pool became more reduced as the exponential phase progressed, even at low culture densities $\left(\mathrm{OD}_{600} \sim 0.2-1.0\right)$ and relatively high oxygen saturation (100 to $\sim 80 \%$ ). In addition, with low-glucose batch cultures, the cellular content of the total pool of oxidized plus reduced species decreased. Concomitant with this decrease, $\mathrm{DMK}_{8}$ and $\mathrm{MK}_{8}$ were synthesized. The presence of $\mathrm{DMK}_{8}$ in cultures that were presumably fully aerobic has also been reported by Shestopalov et al. (1997).

These results justify two important conclusions. First, upon oxygen restriction, E. coli responds on a second-scale response time with synthesis of quinones involved in anaerobic respiration. Second, the total oxygen consumption by the cells may rapidly exceed the oxygen transfer rate from the gas phase to the liquid phase in simple experimental setups such as Erlenmeyer flask cultures in an orbital shaker. This leads to growth conditions that are limited by the availability of oxygen, and therefore results in an increase in the degree of reduction of the components of the respiratory chain. Whether this is the case depends on both the respiratory activity of the cells and the cell density. This may explain the observation that, upon entry into the stationary phase, reoxidation of the UQ pool occurs: non-growing cells have a lower energetic demand and express a lower specific respiration rate (Teixeira de Mattos \& Neijssel, 1997).
As mentioned above, the transition to conditions with low oxygen availability was accompanied by net synthesis of $\mathrm{DMK}_{8}$. It has been shown that the biosynthesis machinery for $\mathrm{MK}_{8}$ is present under aerobic conditions (Shestopalov et al., 1997). The observed fast initiation of net synthesis of this compound suggests that its synthesis is not regulated at the transcriptional or translational level. The data indicate regulation at the post-translational level, i.e. it is determined by the ambient metabolite concentrations and the kinetics of the enzymes of the $\mathrm{MK}_{8}$ biosynthetic pathway.

The technical and experimental difficulties of maintaining oxygen-saturated conditions in batch cultures have been addressed (e.g. Alexeeva et al., 2000). The results presented in this paper describe semiquantitatively the important consequences of inadequate aeration on the physiology of E. coli, and the functioning of its respiratory machinery. They are of importance to research in the field of regulation of redox-related processes, whether they are at the molecular, genetic or physiological level. Currently, we are focusing on a fully quantitative description of the phenomena by making use of chemostat cultures, controlled batch cultures with high oxygen-transfer rates, and highresolution dissolved-oxygen measurements.

\section{ACKNOWLEDGEMENTS}

This work was supported by NWO-ALW project 812.05.004.

\section{REFERENCES}

Alexeeva, S., de Kort, B., Sawers, G., Hellingwerf, K. J. \& Teixeira de Mattos, M. J. (2000). Effects of limited aeration and of the ArcAB system on intermediary pyruvate catabolism in Escherichia coli. $J$ Bacteriol 182, 4934-4940.

Alexeeva, S., Hellingwerf, K. J. \& Teixeira de Mattos, M. J. (2002). Quantitative assessment of oxygen availability: perceived aerobiosis and its effect on flux distribution in the respiratory chain of Escherichia coli. J Bacteriol 184, 1402-1406.

Berg, B. L. \& Stewart, V. (1990). Structural genes for nitrate-inducible formate dehydrogenase in Escherichia coli K-12. Genetics 125, 691702.

Brondijk, T. H., Fiegen, D., Richardson, D. J. \& Cole, J. A. (2002). Roles of NapF, NapG and NapH, subunits of the Escherichia coli periplasmic nitrate reductase, in ubiquinol oxidation. Mol Microbiol 44, 245-255.

Calhoun, M. W. \& Gennis, R. B. (1993). Demonstration of separate genetic loci encoding distinct membrane-bound respiratory NADH dehydrogenases in Escherichia coli. J Bacteriol 175, 3013-3019.

Calhoun, M. W., Newton, G. \& Gennis, R. B. (1991). E. coli map. Physical map locations of genes encoding components of the aerobic respiratory chain of Escherichia coli. J Bacteriol 173, 1569-1570.

Calhoun, M. W., Oden, K. L., Gennis, R. B., de Mattos, M. J. \& Neijssel, O. M. (1993). Energetic efficiency of Escherichia coli: effects of mutations in components of the aerobic respiratory chain. $J$ Bacteriol 175, 3020-3025.

Cole, J. (1996). Nitrate reduction to ammonia by enteric bacteria: redundancy, or a strategy for survival during oxygen starvation?. FEMS Microbiol Lett 136, 1-11. 
Constantinidou, C., Hobman, J. L., Griffiths, L., Patel, M. D., Penn, C. W., Cole, J. A. \& Overton, T. W. (2006). A reassessment of the FNR regulon and transcriptomic analysis of the effects of nitrate, nitrite, NarXL, and NarQP as Escherichia coli K12 adapts from aerobic to anaerobic growth. J Biol Chem 281, 4802-4815.

de Graef, M. R., Alexeeva, S., Snoep, J. L. \& Teixeira de Mattos, M. J. (1999). The steady-state internal redox state (NADH/NAD) reflects the external redox state and is correlated with catabolic adaptation in Escherichia coli. J Bacteriol 181, 2351-2357.

Evans, C. G. T., Herbert, D. \& Tempest, D. W. (1970). The continuous culture of microorganisms. 2. Construction of a chemostat. In Methods in Microbiology, vol. 2, pp. 277-327. Edited by J. R. Norris \& D. W. Ribbons. London: Academic Press.

Geyer, R., Peacock, A. D., White, D. C., Lytle, C. \& Van Berkel, G. J. (2004). Atmospheric pressure chemical ionization and atmospheric pressure photoionization for simultaneous mass spectrometric analysis of microbial respiratory ubiquinones and menaquinones. $J$ Mass Spectrom 39, 922-929.

Hommes, R. W., Simons, J. A., Snoep, J. L., Postma, P. W., Tempest, D. W. \& Neijssel, O. M. (1991). Quantitative aspects of glucose metabolism by Escherichia coli $\mathrm{B} / \mathrm{r}$, grown in the presence of pyrroloquinoline quinone. Antonie Van Leeuwenhoek 60, 373-382.

Lange, H. C., Eman, M., van Zuijlen, G., Visser, D., van Dam, J. C., Frank, J., Teixeira de Mattos, M. J. \& Heijnen, J. J. (2001). Improved rapid sampling for in vivo kinetics of intracellular metabolites in Saccharomyces cerevisiae. Biotechnol Bioeng 75, 406-415.

Lee, P. T., Hsu, A. Y., Ha, H. T. \& Clarke, C. F. (1997). A Cmethyltransferase involved in both ubiquinone and menaquinone biosynthesis: isolation and identification of the Escherichia coli ubiE gene. J Bacteriol 179, 1748-1754.

Neijssel, O. M. \& Teixeira de Mattos, M. J. (1994). The energetics of bacterial growth: a reassessment. Mol Microbiol 13, 172-182.

Otten, M. F. (2001). Structure, function, and regulation of the respiratory network of Paracoccus denitrificans. PhD thesis, Vrije Universiteit Amsterdam, The Netherlands.
Poole, R. K. \& Cook, G. M. (2000). Redundancy of aerobic respiratory chains in bacteria? Routes, reasons and regulation. Adv Microb Physiol 43, 165-224.

Shestopalov, A. I., Bogachev, A. V., Murtazina, R. A., Viryasov, M. B. \& Skulachev, V. P. (1997). Aeration-dependent changes in composition of the quinone pool in Escherichia coli. Evidence of posttranscriptional regulation of the quinone biosynthesis. FEBS Lett 404, 272-274.

Smith, M. W. \& Neidhardt, F. C. (1983). Proteins induced by aerobiosis in Escherichia coli. J Bacteriol 154, 344-350.

Soballe, B. \& Poole, R. K. (1999). Microbial ubiquinones: multiple roles in respiration, gene regulation and oxidative stress management. Microbiology 145, 1817-1830.

Teixeira de Mattos, M. J. \& Neijssel, O. M. (1997). Bioenergetic consequences of microbial adaptation to low-nutrient environments. J Biotechnol 59, 117-126.

Unden, G. (1988). Differential roles for menaquinone and demethylmenaquinone in anaerobic electron transport of E. coli and their fnrindependent expression. Arch Microbiol 150, 499-503.

Van den Bergen, C. W., Wagner, A. M., Krab, K. \& Moore, A. L. (1994). The relationship between electron flux and the redox poise of the quinone pool in plant mitochondria. Interplay between quinoloxidizing and quinone-reducing pathways. Eur J Biochem 226, 1071-1078.

Wallace, B. J. \& Young, I. G. (1977). Role of quinones in electron transport to oxygen and nitrate in Escherichia coli. Studies with a ubiA- menA- double quinone mutant. Biochim Biophys Acta 461, 84-100.

Wissenbach, U., Ternes, D. \& Unden, G. (1992). An Escherichia coli mutant containing only demethylmenaquinone, but no menaquinone: effects on fumarate, dimethylsulfoxide, trimethylamine $\mathrm{N}$-oxide and nitrate respiration. Arch Microbiol 158, 68-73.

Edited by: S. C. Andrews 